\title{
Temperature Stability of Coaxial Cables
}

\author{
K. CzubA* And D. Sikora \\ ISE, Warsaw University of Technology, Nowowiejska 15/19, 05-077 Warsaw, Poland
}

\begin{abstract}
Radio frequency systems developed for large scientific experiments have become increasingly sensitive to phase changes in their subcomponents. Amongst the most important subassemblies there are definitely coaxial cables that provide interconnections between experiment subsystems. It is commonly known that the major source of phase drifts in coaxial cables is their sensitivity to temperature changes. The phenomenon of phase drifts in cables is discussed in this paper. A phase drift measurement setup is described. Samples of cables of various types used in RF system installations have been measured. Measurement results are presented.
\end{abstract}

PACS: $84.40 . \mathrm{Az}$

\section{Introduction}

Modern high-energy physics experiments are large installations spreading over hundreds of meters or even kilometers. Those installations incorporate large quantities of sensitive RF and microwave frequency electronic devices which are used for acceleration and diagnostics of physical particle beam. Amongst the most advanced projects are currently the FLASH [1] and the XFEL [2] facilities located in the scientific institute DESY in Hamburg. Both facilities are dedicated to generation of coherent radiation in ultraviolet and X-ray ranges by use of the so-called free-electron laser (FEL) [2] effect.

The FEL facilities contain multiple RF stations which are used for acceleration of the electron beam. The beam is accelerated by high-gradient electromagnetic fields controlled by sophisticated RF electronics. The field stability and therefore the phase stability of the RF station electronic equipment and subassemblies became critical for the operation of the entire FEL machine. Achieving the required phase regulation of the electric field of $0.1^{\circ}$ at frequency of $1.3 \mathrm{GHz}$ in a large system containing many hundreds of measurement and signal processing channels became a very challenging task. It requires special design and proper selection of the components for accelerating subsystems. Coaxial cables used for transporting high performance measurement and machine synchronization signals are amongst the most important components. Many types of coaxial cables are used in accelerator installations but manufacturers rarely provide the phase stability data.

Besides scientific experiments, the phase stability of coaxial cables is very important for complex microwave devices like radar systems used for military applications. There is few references treating about this prob-

\footnotetext{
* corresponding author; e-mail: kczuba@elka.pw.edu.pl
}

lem, mostly they are commercial papers or application notes like [3]. This paper is devoted to the phase drift characterization of selected coaxial cables used for accelerator subassemblies. Described experiments were performed with modern, commonly used coaxial cables and obtained results should be helpful for selecting proper cable type for a given application. Also described measurement setup could easily be used for characterization of other cable types.

\section{Phase drifts in coaxial cables}

Before the measurements are described a basic explanation of the phase drift phenomenon is needed. Phase drift is a slow change of the phase length of the coaxial cable. The phase length is also known as electrical length which corresponds to the number of wavelengths in the cable at a given frequency.

The phase length can be expressed in units of degrees by the equation

$$
\Phi=\frac{360 f l \sqrt{\varepsilon_{\mathrm{r}}}}{c}
$$

where $f$ is the signal frequency, $l$ is the cable length, $c$ is the light velocity in vacuum and $\varepsilon_{\mathrm{r}}$ is the dielectric constant of the dielectric material filling the space between the center and the outer conductor of the coaxial cable.

Phase length is also frequently expressed in units of time (ns or ps). This interpretation is known as a phase delay and it is the time that it takes the signal to travel through a given length of the cable. The phase delay can be calculated using the following equation:

$$
t_{\mathrm{p}}=\frac{l \sqrt{\varepsilon_{\mathrm{r}}}}{c} .
$$

The phase delay measure is frequency independent. Therefore it is easier to compare phase drifts of the same or various cables at different frequencies. Also many timing specifications for the FEL machines are given in units 
of ps. The phase delay expressed in the time domain units is used in the remnant of this paper.

It was found experimentally that the most important source of phase changes in coaxial cables is their sensitivity to temperature variations. Equations (1) and (2) show that phase drifts can appear with temperature only due to changes of mechanical dimension or the dielectric constant. The final phase drift value is a combined effect of these both factors. The conductor dimensions expand with increasing temperature in a linear and predictable manner [4]. Interestingly, the center conductor expansion usually affects the physical length of the cable. But the expansion of the outer conductor causes change of the mechanical force acting on the dielectric material of the cable (squeezing or loosing) which leads to changes in the density of this material. Finally it causes change of the dielectric constant.

The behavior of the dielectric constant with cable temperature is even more complex and strongly depends on the type of dielectric material used in the cable. One of the most frequently used insulator materials is the poly-tetraflouoro-ethylene (known as PTFE or teflon ${ }^{\mathrm{TM}}$ ). It appears in many standard coaxial cables due to low loss and very good performance over broad range of temperatures. But in temperatures of around $+18^{\circ} \mathrm{C}$ to $19^{\circ} \mathrm{C}$ this material undergoes a molecular phase transition [5] which causes a steep change in the dielectric constant and therefore an abrupt change in the electrical length. This effect is commonly known as the teflon TM "knee" and it can significantly influence the phase stability of the cable in this range of temperatures [6]. The physical basics of this phenomenon are described in [7].

\section{Measurement setup for phase drift characterization}

Measurement setup was prepared for the cable phase drift characterization as shown in Fig. 1. A length of the cable under test was placed in a digitally controlled climate chamber. The chamber allows for flexible programming of temperature profiles, from very fast to slow changes, linear in time.

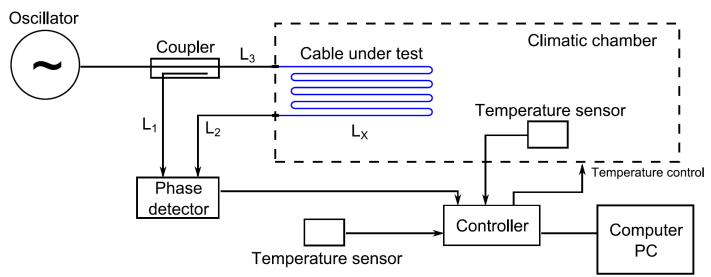

Fig. 1. The phase drift setup.

The phase difference change in the cable was measured by a calibrated phase detector. This device can operate with a wide range of input power levels $(-40 \mathrm{dBm}$ to $+20 \mathrm{dBm}$ ) and therefore a directional coupler was used for splitting the input signal. The coupler with known phase-temperature characteristics was used in the measurements. Test setup cables of known lengths $L_{1}, L_{2}$ and $L_{3}$ were used and the phase drifts of these cables were calibrated out during the data processing. The cables outside the chamber, the phase detector and the directional coupler were thermally isolated. These steps were taken for minimizing the ambient temperatures variations. The climate chamber and the ambient temperatures were measured for the phase drift calculations and for the setup calibration. A high sensitivity thermocouples were used for temperature measurements. All measurement signals were collected by the data acquisition software at the same time in few seconds intervals. The setup was controlled by a software run on a PC, where the temperature profile of the chamber was programmed and the measurement data were processed and stored.

Temperature in the chamber was changed between $+15^{\circ} \mathrm{C}$ and $+40^{\circ} \mathrm{C}$. Such range was selected because the expected ambient temperature within the accelerator environment should not exceed those values. The time of the temperature profile was determined experimentally taking into account the temperature changes in certain areas of the FLASH accelerator. In order to avoid the thermal inertia effects of the cables, the cable under test was warmed up by relatively fast temperatures changes in a wide range and then the setup was temperature stabilized for few hours before applying another measurement temperature changes. Then slow temperature steps were applied with the slopes changing between 3 and $5 \mathrm{~h}$ which resulted to about one degree change per $10 \mathrm{~min}$.

Two sets of measurements were performed for both increasing and decreasing temperature value for characterizing cable's behavior in typical environmental conditions.

Measurements were performed at two signal frequencies: $100 \mathrm{MHz}$ (or $216 \mathrm{MHz}$ ) and $1300 \mathrm{MHz}$. Those frequencies are commonly used in FLASH and XFEL facilities and it was also intended to verify the frequency influence on the phase drift.

The phase difference change was measured by the phase detector calibrated in the units of ps. Results were plot in ps versus temperature and in ppm versus temperature. The second measure is very convenient for calculations of phase drifts for given length of cable.

\section{Calibration and data processing}

Main goal of the calibration was to eliminate the systematic errors in the phase drift measurements. The ambient temperature is not perfectly stable. Therefore three measurements were done to determine the impact of the setup elements on the measurement results due to the ambient temperature changes. Test setups are shown in Fig. 2.

Selected device under test (DUT) was placed in a chamber with variable temperature. The rest of the test bench was placed in another chamber where temperature was stabilized. The first calibration measurement 


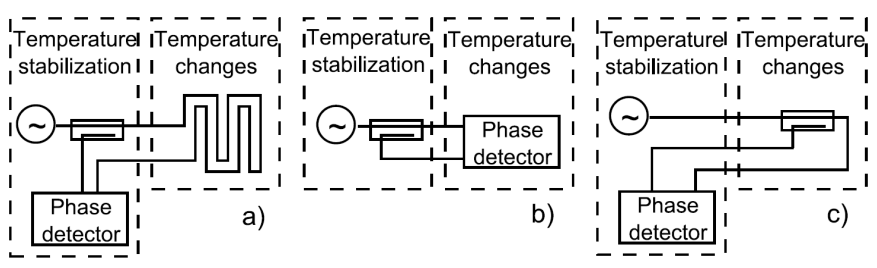

Fig. 2. The calibration setups.

(Fig. 2a) was done for determining the influence of the test setup cables. Data from this measurement was helpful during characterization of the phase detector (Fig. 2b) and the directional coupler (Fig. 2c). It was found that cables have the greatest influence on the test setup performance. Finally by selecting cables with lengths (see Fig. 1) fulfilling Eq. (3), measurement cables influence was minimized

$$
L_{1}=L_{2}+L_{3} .
$$

Temperature coefficients of the directional coupler and the phase detector were recorded.

The next step of the test setup calibration was to find the relationship between the measured phase difference and the phase detector's output voltage. For that purpose two signals of different frequencies (small difference of single $\mathrm{Hz}$ ) were connected to inputs of the phase detector. By this the voltage vs. phase characteristic was achieved in a range of $360^{\circ}$.

During the calibration also random errors were minimized. It was found experimentally that results should be transformed by a low-pass filter (averaging) in the data processing algorithm. This reduced noise effects on the measured data.

After the calibration, as mentioned in Sect. 3, two plots were created: relationship of the phase delay and the phase drift coefficient versus temperature. The phase detector's output voltage was converted to the phase delay (in ps). The phase drift coefficient was calculated as a derivative of the phase delay changes with respect to temperature and expressed in $\mathrm{ppm} /{ }^{\circ} \mathrm{C}$.

\section{Cable selection and measurement results}

Many types of coaxial cables are used in the FEL machine installations. Various cable samples were characterized during this experiment [8]. First results of the work were described in [9]. In this paper extended number of results is provided, together with the preceding ones. Below described are measurement results of five selected cable types: the SS402 which is a standard installation cable at FLASH including teflon ${ }^{\mathrm{TM}}$, the Sucoflex 106 and LL142 who also include teflon ${ }^{\mathrm{TM}}$ but are more phase stable than the SS402, the thin, flexible MRC200-TCOM which is a typical teflon ${ }^{\mathrm{TM}}$-free cable and the $3 / 8^{\prime \prime}$ low loss foam dielectric cable LCF38-50 J which is widely used in a FLASH accelerator installations. This cable also does not contain teflon ${ }^{\mathrm{TM}}$.
Measured were cable samples with lengths of up to $8 \mathrm{~m}$. Results were processed and normalized to $1 \mathrm{~m}$ cable length for easier results comparison. The phase delay versus temperature plots are shown in Fig. 3. In plots from the SS402, the Sucoflex 106 and the LL142 cables, the teflon ${ }^{\mathrm{TM}}$ knee effect is clearly visible as relatively steep phase change in the temperature range between $+15^{\circ} \mathrm{C}$ and $+22^{\circ} \mathrm{C}$. For temperatures above $+23^{\circ} \mathrm{C}$ the teflon $^{\mathrm{TM}}$ transition is over and interestingly, the SS402 presents almost linear phase change with temperature, the Sucoflex 106 and the LL142 phase plots are almost flat in this temperature range. Most probably the SS402 behavior corresponds directly to the thermal conductor expansion and in the Sucoflex 106 and the LL142 the thermal expansion effect is compensated by another effect like change of the dielectric pressure.

Another very important observation is the hysteresis in the measurement plots. Due to different behavior of the molecular transition when increasing and when decreasing temperature various signal phase transitions are measured. This effect causes difficulties in numerical compensation of phase drifts as one would need to record the direction of temperature changes.

It is also interesting that observed phase changes significantly depend on the signal frequency. We could not find the explanation for this phenomenon but most probably it is connected to some properties of the dielectric material.

The phase versus temperature plot of the MRC200-TCOM cable is shown in Fig. 3c. The teflon ${ }^{\mathrm{TM}}$ knee effect is not visible and there is also no hysteresis. The slope of the line is almost constant so most probably we observe only the effect of thermal expansion. As mentioned above, there is also no significant difference in the plot for both measurement frequencies which confirms the statement that the presence of teflon ${ }^{\mathrm{TM}}$ causes the frequency dependence of phase drift value.

The phase versus temperature plot of the $3 / 8^{\prime \prime}$ cable is shown in Fig. 3e. The hysteresis and the teflon ${ }^{\mathrm{TM}}$ knee effect is also not visible like in the MRC200-TCOM cable. At the temperature of $\approx 25^{\circ} \mathrm{C}$ (at $1300 \mathrm{MHz}$ ) this cable is ultra phase stable with almost 0 ps of phase change. In the entire measurement temperature range very low phase changes are achieved not exceeding $0.16 \mathrm{ps} /{ }^{\circ} \mathrm{C}$, which is of order of magnitude better result than for the other cable types.

Measurement results for the five selected cables expressed in units of $\mathrm{ppm} /{ }^{\circ} \mathrm{C}$ are shown in Fig. 4. In three teflon ${ }^{\mathrm{TM}}$ based cables the temperature coefficient becomes almost constant for temperatures above $23^{\circ} \mathrm{C}$. Interestingly is that the temperature coefficients of the Sucoflex 106 and the LL142 are almost zero in this range. Therefore, in a "warm" environment, these cables can be used for building almost drift-free connections. For broader temperature ranges and for applications, where use of relatively thick cables is possible, the 3/8" LCF38-50 J cable is the best option. In Fig. 4e the phase drift coefficient values are shown and they are very 

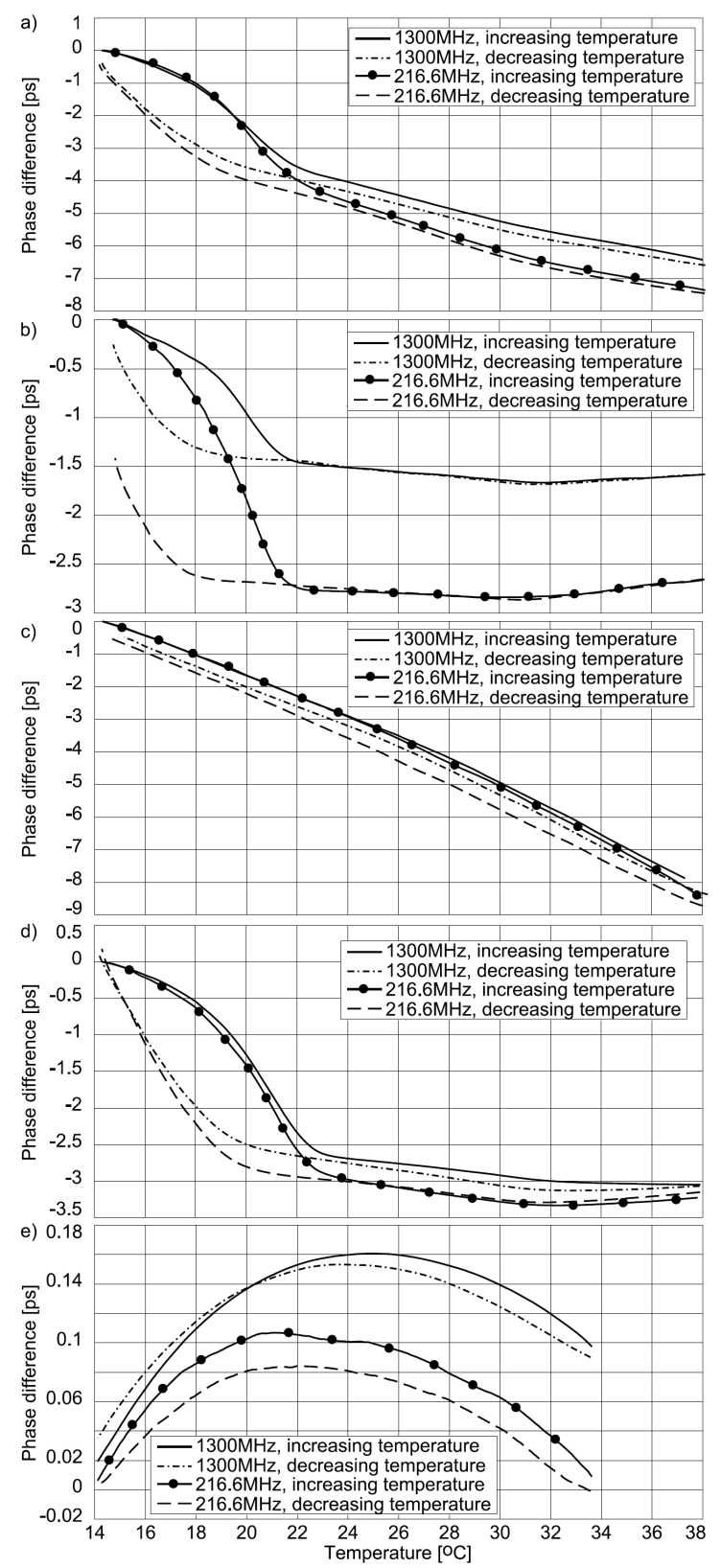

Fig. 3. The phase change versus temperature of the cables: (a) SS402, (b) sucoflex 106, (c) MRC200-TCOM, (d) LL142, (e) LCF38-50J - 3/8" cable.

small (in range $\pm 6 \mathrm{ppm} /{ }^{\circ} \mathrm{C}$ ) in wide temperature range and reach zero at about $24^{\circ} \mathrm{C}$.

Plots in Fig. 4a and b show also that the fast phase transition (teflon ${ }^{\mathrm{TM}}$-knee) appears at lower temperatures when the temperature is decreasing. For increasing temperatures this effect occurs as described above, around $+19^{\circ} \mathrm{C}$.

The MRC200-TCOM cable exhibits almost constant temperature coefficient but relatively large $(-60$ to $-90 \mathrm{ppm} /{ }^{\circ} \mathrm{C}$ ) comparing to the flat portion of the plot in Fig. 4b.

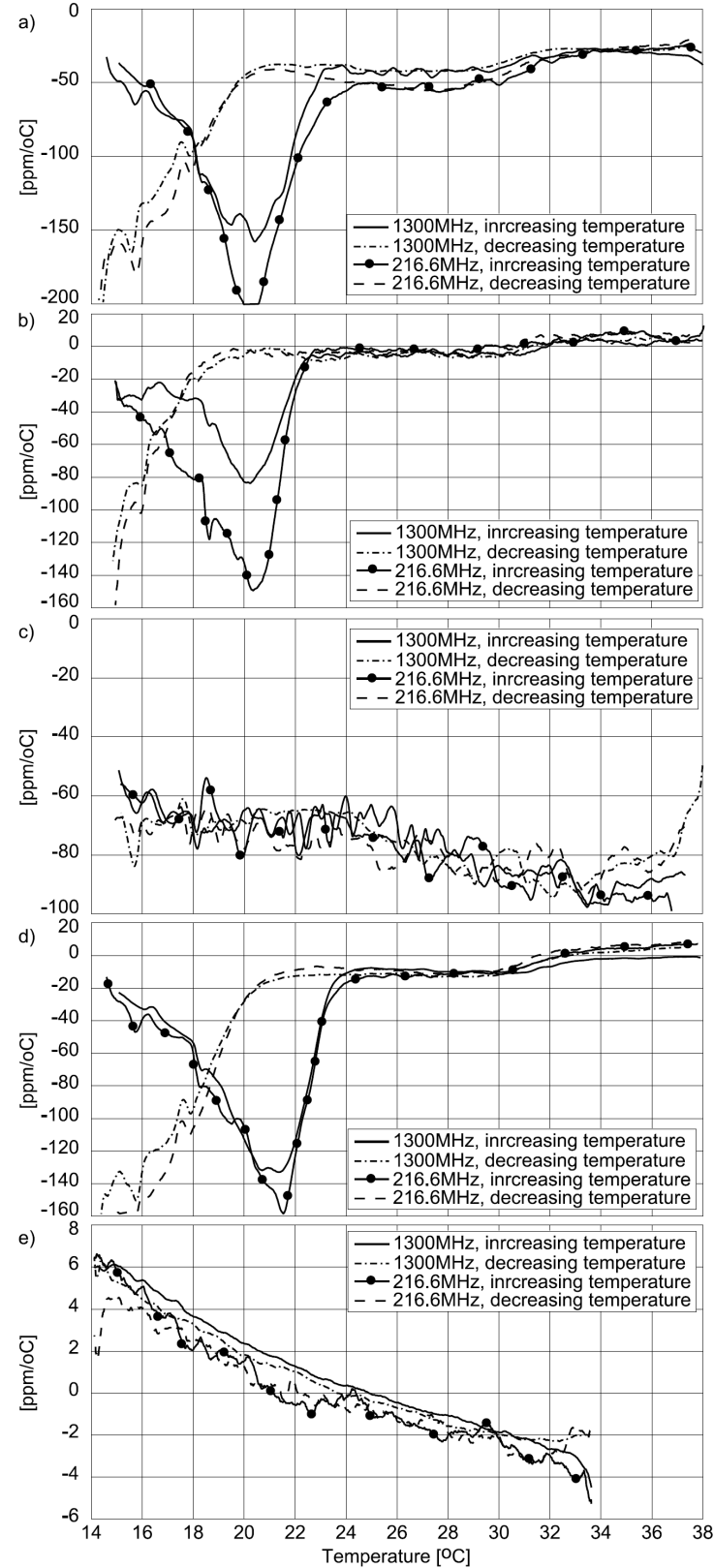

Fig. 4. The phase coefficient versus temperature of the cables: (a) SS402, (b) sucoflex 106, (c) MRC200-TCOM, (d) LL142, (e) LCF38-50J - 3/8" cable.

\section{Summary}

Measurements performed with the prepared test setup show complex relationship of coaxial cable phase drifts to the temperature changes. The standard cables with the PTFE dielectric material exhibit the teflon ${ }^{\mathrm{TM}}$ knee phenomenon and hysteresis which may cause difficulties with prediction and compensation of phase drifts when operating cables in temperatures between $15^{\circ} \mathrm{C}$ and $22^{\circ} \mathrm{C}$. The phase vs. temperature coefficient of the PTFE-free cables does not change abruptly with temperature. The selection of proper cable for low phase drift application may 
strongly depend on the operating temperature range. If the cable is supposed to operate in environment temperatures below $22^{\circ} \mathrm{C}$ and no needs for flexible cable, the teflon free cable should be selected. Otherwise, better choice will be one of the PTFE based cable types. Significant improvement of phase stability can be achieved by applying temperature stabilization on the cables. The plots described in this paper may be very helpful for this purpose.

\section{Acknowledgments}

We would like thank the Elspec GmbH company for providing us professional cable samples.

The research leading to these results has received funding from the European Commission under the EuCARD FP7 Research Infrastructures grant agreement no. 227579

\section{References}

[1] http://flash.desy.de/ .

[2] The European X-ray Free-Electron Laser Technical Design Report, DESY XFEL Project Group, Hamburg, July 2007.
[3] Coaxial Cables - Phase Matching and Tracking Guidelines, Tyco Electronics Application Note, AN3029.

[4] Understanding Phase versus Temperature Behavior "The Teflon ${ }^{\mathrm{TM}}$ Knee", Micro-Coax Application Note.

[5] Times Microwave Systems, Current Innovations in Phase Stable Coaxial Design, Microwave Product Digest.

[6] R. Schwartz, Microwave J. 52, 16 (Supplement) (2009).

[7] Satish K. Ghawan, Understanding Effect of Teflon Room Temperature Phase Transition on Coax Cable Delay in Order to Improve the Measurement of TE Signals of Deuterated Polarized Targets, J.W. Gibbs Laboratory, Yale University, New Haven, CT, 06511.

[8] D. Sikora, M.Sc. Thesis, Warsaw University of Technology, 2010, p. 25, (in Polish).

[9] K. Czuba, D. Sikora, in: Proc. 18th Int. Conf. on Microwave Radar and Wireless Comunnication (MIKON 2010), Vilnius (Lithuania), Ed. B. Levitas, Jusida, Vilnius 2010, p. 388. 